\title{
DESENVOLVIMENTO DE SOFTWARE PARA A OTIMIZAÇÃO DA PRODUÇÃO HORÁRIA DE EQUIPAMENTOS EM OPERAÇÓES DE TERRAPLENAGEM
}

\author{
J. G. C. Azevedo ${ }^{1}$; D. F. Vicentini² \\ 1,2 Universidade Federal do Paraná, Av. Cel. Francisco H. dos Santos, 210 \\ Jd. das Américas, CuritibalPR, CEP 81530-000 \\ joao.g-azevedo@uol.com.br $r^{l}$ vicentini@ufpr.br ${ }^{2}$
}

\begin{abstract}
Resumo: Ferramentas simples são utilizadas há milhares de anos e, com o tempo passaram a se tornar mais robustas. Com o advento dos motores, ferramentas complexas tornaram-se máquinas. Toda essa evolução ocorreu com o mesmo objetivo: aumentar e facilitar a qualidade e quantidade dos serviços realizados. Todos os projetos de construção civil exigem diferentes equipamentos, cada qual para diferentes tipos de aplicaçóes. A dependência humana para com as máquinas vem crescendo cada vez mais, visto que sua facilidade e versatilidade têm se tornado requisitos indispensáveis. O presente trabalho pretende facilitar a utilização de cálculos de produção horária, através da implementação de um algoritmo com interface gráfica moderna e amigável, permitindo realizar sistematicamente os cálculos e apresentar soluçóes em termos de combinaçóes de equipes mecânicas. $\mathrm{O}$ algoritmo, implementado em Visual Basic, utiliza e facilita algumas etapas de cálculo unitário de serviços de acordo com os manuais do DNIT. Através do programa sáo calculados dados de produçáo horária, prazos e custos dos serviços, permitindo encontrar a equipe ótima, sob determinados parâmetros. $\mathrm{O}$ algoritmo foi aplicado em exemplos disponíveis na literatura para comparação e produziu resultados satisfatórios, gerando gráficos que auxiliam nas decisóes do usuário. $\mathrm{O}$ algoritmo foi inicialmente idealizado para uso didático, porém seu uso permitirá maior agilidade na tomada de decisóes por parte de empresas e organismos.
\end{abstract}

Palavras-chave: Equipamentos de construção, Custos, Produtividade, Eficiência, Otimização de equipes.

\begin{abstract}
Simple tools have been used for thousands of years, becoming stronger with the time. With the advent of engines, complex tools became machines. All this evolution occurred with the same objective: to increase and facilitate the quality and quantity of services performed. All construction projects require different equipment, each one for different types of applications. Human dependence on machines has been growing more and more since its facility and versatility have become essential requirements in projects. The present work intends to facilitate the use of hourly production calculations, through the implementation of an algorithm with a graphical modern and user interface, allowing the systematic perform of calculations and present solutions of combinations of mechanical teams. The algorithm was implemented in Visual Basic environment. It uses and facilitates some steps of unit cost of services, according to DNIT standards. The program generates production, time and cost data, allowing to find the optimal team, under certain parameters. The algorithm was applied in examples available in the literature, presenting satisfactory results. It also generates graphics for decisions which will give support to the user. The algorithm was initially idealized for didactic applications, nevertheless its use will also allow faster decision-making for companies and organizations.
\end{abstract}

Keywords: Construction equipment, Costs, Production, Efficiency, Team optimization. 


\section{Introduçáo}

Construçôes de estradas, ferrovias, aeroportos, edificaçóes, obras de infraestrutura, ou até mesmo um pequeno conjunto residencial exigem, em sua maioria, operaçóes prévias de terraplenagem, para posterior execução do projeto a ser implantado. Assim, pode-se dizer que todas as obras de Engenharia Civil de grande, médio, ou pequeno porte exigem trabalhos de movimentação de terras [1].

Os serviços de terraplenagem e pavimentação, por lidarem com a movimentação de milhares de toneladas de material e um grande número de equipamentos pesados, requerem uma atenção especial por parte dos construtores e dos órgãos contratantes. Assim, é necessário que a movimentação dos materiais entre cortes, jazidas e aterros seja feita de forma racional para que se consiga uma redução no custo das obras [2].

A necessidade e a dependência de equipamentos de construção pesados têm crescido com o tamanho e a complexidade dos projetos. O desenvolvimento de equipamentos automatizados para movimentação de terras, escavação e acabamento, entretanto vem ocorrendo somente desde os dois últimos séculos [3].

Ao analisar a história dos equipamentos, é notável observar que as operaçôes e os princípios mecânicos para a maioria deles são basicamente os mesmos de suas primeiras versóes, criadas há séculos atrás. Ferramentas simples eram normalmente utilizadas para construir um ambiente melhor para se viver. Elas melhoravam a velocidade, a quantidade e a qualidade das atividades que eram realizadas pelo homem. Com o decorrer dos séculos, ferramentas mais complexas começaram a ser pensadas. Passou-se a utilizar outras forças, que não a do homem, para a realização de atividades, entre elas estão a força das águas e dos ventos, através das rodas d'água e moinhos de vento.

O maquinário pesado usado atualmente é resultado de grandes melhorias baseadas na necessidade de se trabalhar com maior eficiência, efetividade e segurança. Basicamente, os desenvolvimentos existentes nesta área foram baseados em redução nos custos, aumento na velocidade e melhorias na segurança [3].

Tendo em vista a grande importância dos equipamentos de construção civil para a terraplenagem, foi traçado como objetivo buscar uma forma de melhorar essas atividades. Uma das formas mais eficazes é trazendo mais efetividade nas escolhas e, com isso, melhorar a produção e consequentemente os prazos e custos.

Durante a fase de construção, a seleção correta dos equipamentos tem sido um fator importante para o sucesso de qualquer projeto [4]. No entanto, em grande parte dos casos, o responsável pelo projeto, através de sua experiência, escolhe intuitivamente os tipos, quantidades, capacidades entre outras características necessárias de tais equipamentos, sem o uso de métodos apropriados, incidindo diretamente na eficiência da produção. 
O presente trabalho pretende facilitar a utilização de tais cálculos, através do desenvolvimento de um software com interface gráfica moderna e amigável, ou seja, um programa que realize sistematicamente os cálculos, agilizando e auxiliando seu usuário na decisão por um equipamento ou equipe mecânica com vistas à maior produtividade e eficiência, redução de custos e prazo.

\section{Metodologia}

Buscando cumprir com os objetivos do trabalho, a seguir são apresentadas as delimitações do estudo, a metodologia utilizada, os procedimentos seguidos, o software desenvolvido e outros itens que foram testados.

Durante o desenvolvimento deste trabalho, verificou-se uma grande diversidade de equipamentos, em diferentes áreas, portanto houve a necessidade de delimitação do escopo de estudo. Assim, foram identificadas três principais áreas de atuação dos equipamentos na construção: terraplenagem, pavimentação e drenagem [5], das quais são apresentados (Tabela 1) um resumo das operaçóes e composição de seus custos unitários:

Tabela 1 - Operaçóes e composição dos custos unitários para as principais atividades na construção [5].

\begin{tabular}{lcccc}
\hline & Materiais & Máo de obra & Equipamentos & Transporte \\
\hline Terraplenagem & $18 \%$ & $15 \%$ & $52 \%$ & $15 \%$ \\
Pavimentaçáo & $74 \%$ & $3 \%$ & $19 \%$ & $4 \%$ \\
Drenagem & $69 \%$ & $23 \%$ & $4 \%$ & $4 \%$ \\
\hline
\end{tabular}

De acordo com a Tabela 1, é possível observar que a terraplenagem é a atividade cujo custo unitário é mais influenciado pelo uso de equipamentos (ultrapassando 50\% do custo da atividade), sendo esta a razão de sua escolha. No âmbito desta atividade, alguns dos equipamentos mais comuns foram selecionados: pá carregadeira, caminhão basculante, escavadeira hidráulica, rolo compactador, motoniveladora, e caminhão espargidor/irrigador.

Para a realização do cálculo do custo unitário do serviço, é também calculado o custo operacional dos equipamentos, em que incidem depreciação, juros, manutenção, material e mão de obra. Essa etapa foi substituída pela utilização do custo horário do equipamento, ou seja, foi considerado que o usuário já possui, em seus históricos, o custo de seu equipamento, ou poderá ser realizada a locação do equipamento, prática muito usual em obras de terraplenagem. Neste trabalho serão utilizados valores de aluguel, obtidos através da startup COLLOC, a fim de se obter de resultados mais práticos. 


\subsection{Composiçáo de custos}

O objetivo do software criado é facilitar a utilização dos procedimentos encontrados em [6, 7 , 8]. Em [6], o procedimento em que a produção horária dos equipamentos é calculada, é chamado de método teórico. De acordo com o serviço a ser realizado, os equipamentos são escolhidos e combinados, para então identificar as variáveis intervenientes, e assim calcular suas produçôes horárias individuais e definir a produção horária de toda a equipe.

Para implementar esta etapa, foi utilizada a planilha de produçóes das equipes mecânicas, como no exemplo extraído de [7], apresentado na Fig. 1:

\begin{tabular}{|c|c|c|c|c|}
\hline \multicolumn{2}{|c|}{ CODIGO } & \multicolumn{2}{|l|}{ SERVIÇO } & \multirow[t]{2}{*}{ UNIDADE } \\
\hline & \multicolumn{2}{|c|}{$\begin{array}{c}\text { VARIÄVEIS } \\
\text { INTERVENIENTES } \\
\end{array}$} & EQUIPAMENTOS & \\
\hline & & UNIDADE & & \\
\hline a & AFASTAMENTO & & & \\
\hline $\mathrm{b}$ & CAPACIDADE & & & \\
\hline $\mathrm{c}$ & CONSUMO (QUANTIDADE) & & & \\
\hline d & DISTANCIA & & & \\
\hline e & ESPAÇAMENTO & & & \\
\hline$f$ & ESPESSURA & & & \\
\hline$g$ & FATOR DE CARGA & & & \\
\hline $\mathrm{h}$ & FATOR DE CONVERSÁOO & & & \\
\hline$i$ & FATOR DE EFICIENCIA & & & \\
\hline $\mathrm{j}$ & LARGURA DE OPERAÇAO & & & \\
\hline 1 & LARGURA DE SUPERPOSIÇÃO & & & \\
\hline $\mathrm{m}$ & LARGURA UTTL & & & \\
\hline $\mathrm{n}$ & NUUMERO DE PASSADAS & & & \\
\hline$\circ$ & PROFUNDIDADE & & & \\
\hline$p$ & $\begin{array}{|ll|}\text { TEMPO FIXO } & \text { (CARGA, } \\
\text { DESCARGA E MANOBRA) }\end{array}$ & & & \\
\hline q & TEMPO PERCURSO (IDA) & & & \\
\hline$r$ & TEMPO DE RETORNO & & & \\
\hline 5 & TEMPO TOTAL DE CICLO & & & \\
\hline$t^{t}$ & VELOCIDADE (IDA) MEDIA & & & \\
\hline u & VELOCIDADE RETORNO & & & \\
\hline & & & & \\
\hline & & & & \\
\hline & OBSERVAÇŐES & & FÓRMULAS & \\
\hline$\overline{\mathrm{PRC}}$ & DUÇÃO HORÁRIA & & & \\
\hline NÚN & IERO DE UNIDADES & & & \\
\hline UTIL & IZAÇÄO OPERATIVA & & & \\
\hline $\begin{array}{l}\text { UTII } \\
\text { IMP } \\
\end{array}$ & $\begin{array}{l}\text { IZAÇÃO } \\
\text { RODUTIVA }\end{array}$ & & & \\
\hline PRC & DUÇÄO DA EQUIPE & & & \\
\hline & $\begin{array}{l}\text { MT/DNIT - Departament } \\
\text { Infra-Estrutura de Tr }\end{array}$ & $\begin{array}{l}\text { to Nacional de } \\
\text { ransportes }\end{array}$ & $\begin{array}{r}\text { PRODUÇÃO D, } \\
\text { MECÂN }\end{array}$ & \\
\hline & SISTEMA DE CUSTOS RODOVV & & & \\
\hline
\end{tabular}

Figura 1 - Planilha de produção das equipes [7]. 
Para preenchimento desta planilha, as fórmulas de cálculo para obtenção das produções horárias, foram obtidas a partir de $[8,9]$, e são apresentadas na Tabela 2 , sendo as variáveis: $\mathrm{C}$ = capacidade volumétrica $\left(\mathrm{m}^{3}\right), \mathrm{f}=$ fator de conversão de volume, $\mathrm{f} 1$ = fator de carga, $\mathrm{E}$ = eficiência do equipamento, $\mathrm{T}$ = tempo de ciclo completo (minutos), $\mathrm{L}=$ largura útil (metros), $\mathrm{v}=$ velocidade $(\mathrm{m} / \mathrm{min}), \mathrm{n}$ = número de passadas, $\mathrm{D}=$ distância (metros), e = espessura (metros), $\mathrm{c}=$ consumo $\left(\mathrm{em} \mathrm{l} / \mathrm{m}^{3}\right)$.

Tabela 2 - Fórmulas de cálculo para produção horária [8, 9].

\begin{tabular}{cc}
\hline Equipamento & Produçáo horária $\left(\mathbf{m}^{3} / \mathbf{h}\right)$ \\
\hline Pá carregadeira & $P h=\frac{60 * C * f * f 1 * E}{T} m^{3} / h$ \\
Rolo compactador & $P h=\frac{60 * e * E * L * v}{n} m^{3} / h$ \\
Caminhão basculante & $P h=\frac{60 * C * E}{T} m^{3} / h$ \\
Escavadeira hidráulica & $P h=\frac{60 * C * f * f 1 * E}{T} m^{3} / h$ \\
Motoniveladora & $P h=\frac{60 * D * e * E * L}{n * T} m^{3} / h$ \\
Caminhão espargidor & $P h=\frac{60 * \leftarrow * E}{c * T} m^{3} / h$ \\
\hline
\end{tabular}

A planilha de custo unitário de serviço também sofreu reduçôes, sendo utilizada apenas a parte A (Fig. 2):

\begin{tabular}{|c|c|c|c|c|c|c|}
\hline \multicolumn{7}{|c|}{ CUSTO UNITARIO DO SERVIÇO } \\
\hline SERVIÇO: & & & & & & UNIDADE: \\
\hline \multirow{2}{*}{ EQUIPAMENTO } & \multirow{2}{*}{ QUANT } & \multicolumn{2}{|c|}{ UTILIZACAÁO } & \multirow{2}{*}{\multicolumn{2}{|c|}{ CUSTOOPERACIONAL }} & \multirow{2}{*}{ CUSTO HOPÁRIO } \\
\hline & & PROD. & IMPROD & PROD. & & \\
\hline \multirow{4}{*}{1} & 2 & 2 & 4 & 5 & 6 & 7 \\
\hline & & & & & & \\
\hline & & & & & & \\
\hline & & & & $\mathbf{A}$ & & \\
\hline & & & & & & \\
\hline & & & & & & \\
\hline & & & & & $\overline{\mathrm{OT}}$ & \\
\hline
\end{tabular}

Figura 2 - Planilha de custo unitário do serviço. Em detalhe, a parte A [7].

Todos os itens da parte A serão calculados, exceto o custo operacional, que será substituído pelo custo de aluguel da máquina, como mencionado acima. 
A partir da produção horária da equipe e da quantidade de material do serviço, o tempo necessário para sua realização é calculado.

\subsection{Desenvolvimento do software}

Para criação do software foi utilizada a linguagem de programação Visual Basic, derivada do Beginner's All-purpose Symbolic Instruction Code (BASIC), escolhida pela facilidade de aprendizado e por apresentar uma interface de usuário interessante, permitindo adicionar uma série de ferramentas dinâmicas e menus iterativos (check-box, listas, botôes, geração de gráficos).

Microsoft Visual Studio Community foi o software utilizado juntamente/acoplado ao Visual Basic, sendo ambos de livre utilização para programas com linguagem aberta, um exemplo da tela principal do programa pode ser visto na Fig. 3. O manual do usuário foi criado através do programa Dr.Explain.



Figura 3 - Interface do programa Microsoft Visual Studio.

As etapas realizadas na implementação podem ser resumidas através de um fluxograma, mostrando o processo de funcionamento do programa, e indicado na Fig. 4. 


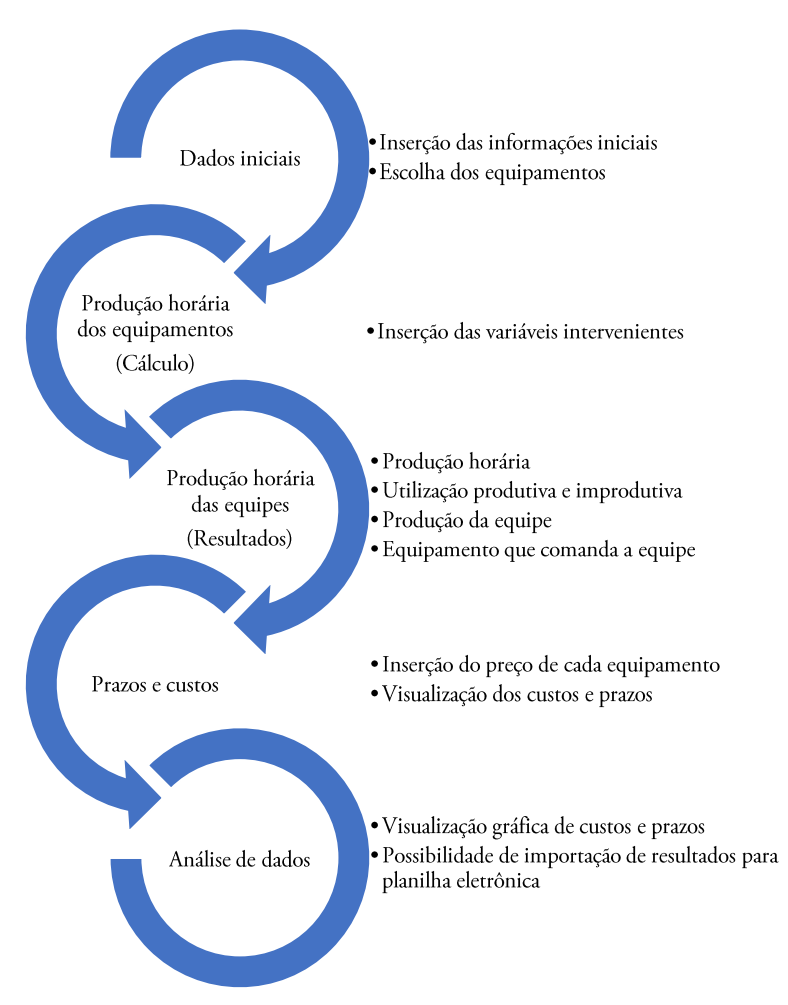

Figura 4 - Fluxograma de funcionamento do software.

\section{Resultados}

O presente trabalho permitiu gerar um programa em interface windows, capaz de calcular a produção horária de equipamentos e equipes, capaz de realizar uma análise comparativa entre estes, além do custo final da operação e o tempo do serviço.

Nas Fig. 5 e Fig. 6 são mostradas como exemplo duas telas do programa, geradas a partir de um exemplo retirado da literatura [8], onde pede-se que seja calculado o prazo de execução de um serviço de transporte de 14160,0 $\mathrm{m}^{3}$ de areia solta, empregando-se cinco caminhóes basculantes e uma pá carregadeira de pneus (pág. 65). Os gráficos gerados podem auxiliar na decisão, comparando os custos de equipamentos de uma mesma equipe, e assim ajudar a identificar equipamentos onerosos. Deste modo, na Fig. 5, é apresentado um gráfico comparativo entre os custos de uma equipe formada por uma pá carregadeira e cinco caminhóes basculantes, realizada através do dimensionamento com a equipe disponível. Para comparação foi selecionada uma equipe de pá carregadeira e caminhão, porém com o dimensionamento ótimo (obtido com a utilização do software), o que resultou no número de sete caminhốes basculantes. 


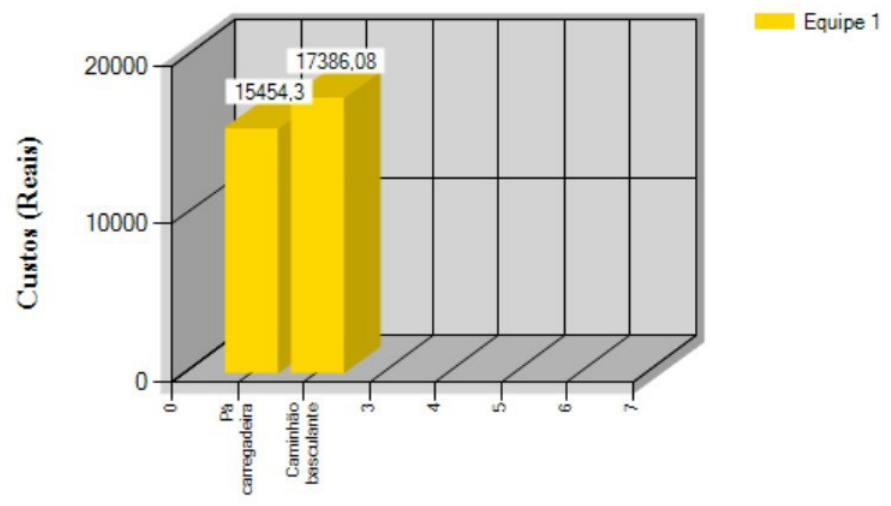

Equipamentos

Figura 5 - Custo dos equipamentos para uma equipe.

$\mathrm{Na}$ Fig. 6 é apresentado um gráfico emitido pelo software com o comparativo entre as duas equipes, selecionadas para a mesma atividade, porém com uma combinação diferente de equipamentos: 1) Uma pá-carregadeira e cinco caminhóes, equipe disponível (o número de equipamentos é selecionado pelo usuário) e 2) Uma pá carregadeira e sete caminhôes, equipe ótima (onde o número de caminhóes é calculado pelo software).

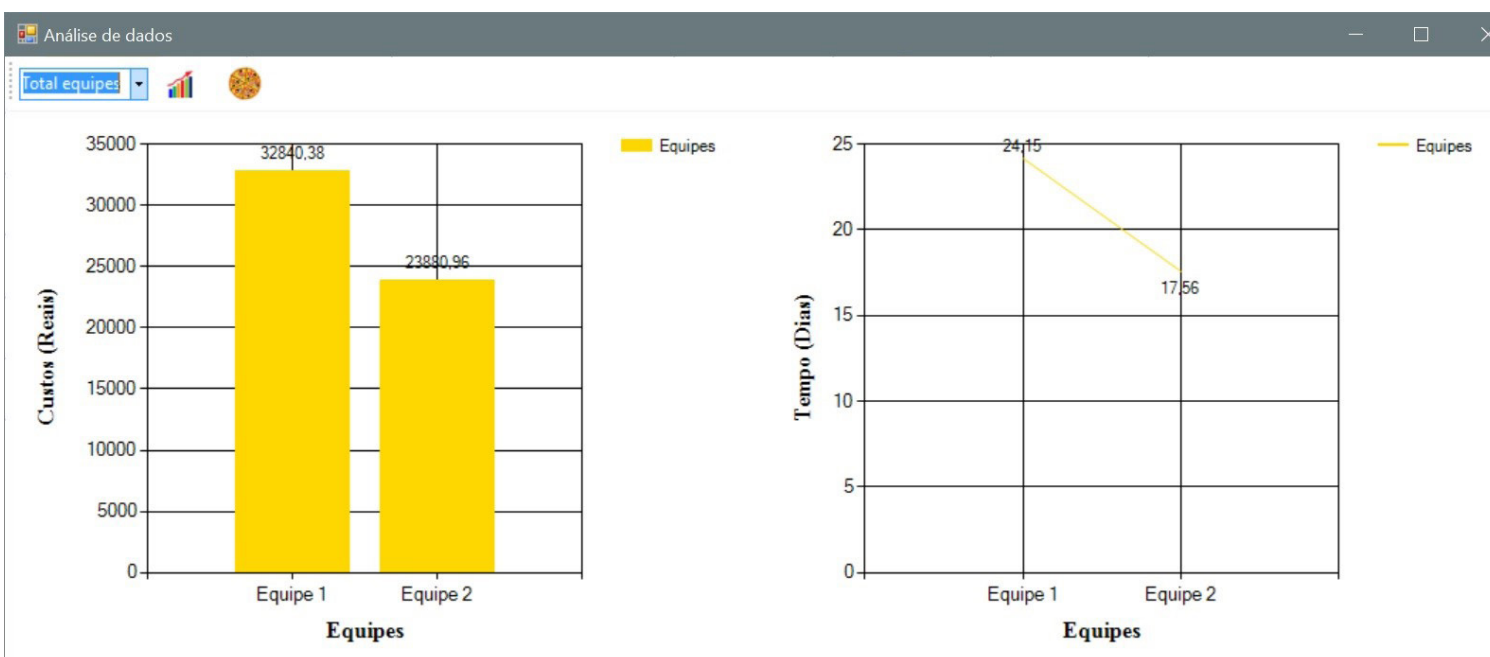

Figura 6 - Custo e prazos de equipes.

Assim, de acordo com este exemplo, a equipe 2 apresentou melhor produtividade e menores custos, com sete dias a menos de serviço que a equipe 1 . Isso se deve ao fato de o equipamento que 
limita a produção horária ser a pá carregadeira ao invés do caminhão, como na equipe 1 . Por ter uma produção maior em relação ao caminhão a atividade realizada pela equipe 2, em que a pá carregadeira comanda, durará menos tempo, e isso refletirá nos custos, ou seja, com mais equipamentos reduziu-se o tempo e houve redução nos custos totais da operação conjunta.

\section{Conclusóes}

O desenvolvimento do presente estudo possibilitou a criação de um software que auxilia a escolha de equipamentos em operaçôes de terraplenagem. Basicamente, com ele é possível comparar equipes e obter maior eficiência na produção, rapidez e redução de custos.

De um modo geral, a maioria dos equipamentos não obedece a um critério rigoroso de escolha (em função da produtividade da equipe), seja por razôes de disponibilidade ou por acreditar ser mais prático e rápido. Esse tipo de atitude pode promover atrasos em obras e comprometer a eficiência das equipes formadas.

O programa desenvolvido facilita um trabalho que pode ser realizado através de várias tabelas e fórmulas, aumentando assim a utilização dessas metodologias, já que os cálculos são realizados sistematicamente pelo programa, ao invés da necessidade de se produzir planilhas e preencher onerosas tabelas. Os recursos de geração automática de gráficos também criam outra facilidade para a análise imediata de dados, facilitando seu uso.

O usuário ainda pode exportar os dados inseridos e realizar cálculos rapidamente. Os resultados obtidos, com exceção dos gráficos, podem ser ainda exportados para uma planilha eletrônica, e assim manejá-los da maneira desejada.

Pretende-se ainda utilizar o software para fins acadêmicos, o que proporcionará uma ferramenta didática para verificaçóes e estudos de casos que se façam necessários, através da disciplina Equipamentos de Construção e Conservação e Infraestrutura Viária, além de promover o interesse por parte dos alunos no assunto, abrindo várias possibilidades, como novas pesquisas na área, aplicação em casos práticos para constatar a efetividade desses métodos, ou até mesmo expansão da linha de programação com inclusão de novas funçôes, atividades e equipamentos. O software será disponibilizado como software livre e também poderá ser utilizado por engenheiros e empreiteiros que desejarem uma maior produtividade e eficiência em suas obras de terraplenagem.

\section{Referências}

[1] RICARDO, H. D.; CATALANI, G. Manual prático de escavaçáo: terraplenagem e escavaçáo de rocha. São Paulo: PINI, 2007.

[2] LIMA, R. X.; NOBRE JÚNIOR, E. F. Logística Aplicada à construçáo rodoviária. Reshearch Gate, 2003.

[3] GRANSBERG, D. D.; POPESCU, C. M.; RYAN, R. Construction Equipment Management for Engineers, Estimators, and Owners. CRC Press, 2006. 
[4] WARRIS, M.; SHAIR LIEW, M.; FARIS KHAMIDI, M.; IDRUS, A. Criteria for the selection of sustainable onsite construction equipment. International Journal of Sustainable Built Environment 3, p. 96-110, 2014.

[5] PEDROZO, L. G. Custos da infraestrutura rodoviária - Análise e Sistematização. Rio Grande do Sul, 2001. Disponível em: <http://hdl.handle.net/10183/2809>. Acesso em: 3 nov. 2017.

[6] BRASIL, DEPARTAMENTO NACIONAL DE INFRAESTRUTURA DE TRANSPORTES. Manual de custos rodoviários. Rio de Janeiro: 3. ed., 2003.

[7] PEREIRA, D. M.; FRANCO, E. J.; RATTON, E.; BLASI, G. F.; BERNARDINIS, M. A. P.; KUSTER FILHO, W. Composiçáo de custos para obras rodoviárias. Curitiba, 2015. Disponível em: <http://www.dtt.ufpr.br/ TransportesA/Arquivos/Apostila_ComposicaoCustos-2008.pdf>. Acesso em: 6 nov. 2017.

[8] JAWORSKI, T. Equipamentos para escavaçáo - compactaçáo e transporte. Curitiba, 2015. Disponível em: <http://www.tecnologia.ufpr.br/portal/dtt/tt047-equipamento-de-construcao-e-conserva/>. Acesso em: 6 nov. 2017.

[9] GUIMARÃES, N. Equipamentos de construçáo e conservaçáo. Curitiba: Ed. UFPR, 2001. 